\title{
INTEGRATED PRODUCT DATA MANAGEMENT BASED ON A UNIFORM USER INTERFACE
}

\author{
Wolfram Wöß \\ Institute for Applied Knowledge Processing (FAW) \\ Johannes Kepler University Linz \\ Altenberger Straße 69, A-4040 Linz, Austria \\ Tel: +4373224689589 Fax: +4373224689308 \\ e-mail:wwoess@faw.uni-linz.ac.at
}

Today many enterprises are forced to produce products which fulfill individual customer requirements. This situation results in an exploding number of variant parts of a product again resulting in a huge number of product data. To meet these new requirements, many enterprises introduced powerful CIM systems. Normally, this leaded to heterogeneous EDP concepts which are characterized by autonomous CIM components, each with its own database(s) and with a specific user interface.

This paper illustrates how integrated rule-based product data management on the one hand significantly reduces the amount of product data that has to be administrated and how integrated product data management on the other hand allows to automate data exchange between several CIM components. The presented approach also includes a powerful and uniform user interface for product data management.

\section{INTRODUCTION}

Today many enterprises are forced to produce products which fulfill individual customer requirements. This situation results in an exploding number of variant parts of a product again resulting in a huge number of product data (Breitenstein 1994, Poensgen 1994). To meet these new requirements, many enterprises introduced powerful CIM (computer integrated manufacturing) systems. Normally, this leaded to heterogeneous EDP (electronic data processing) concepts which are characterized by autonomous CIM components, each with its own specialized database(s) and with a specific user interface.

Due to this situation, now many networked CIM enterprises are confronted with the following problem areas:

- The data representation of individual customer production results in a large number of different final products in combination with a very large number of bills of material and routing plans which are necessary for the production (planning) process. The maintenance of such a large number of product data with 
conventional methods and software systems is often made too expensive or sometimes even impossible (Anderl 1997, Bass 1996, Thomas 1992).

- To keep all individual databases in a consistent state, it is necessary to exchange (product) data between several databases. Especially business oriented systems and technical systems of an enterprise are characterized by different structural views on the same product data. The consequence is, that interoperability between different CIM components is often impossible and data exchange has to be done manually.

- Planning algorithms and knowledge about bill of material processing, operations planning etc. are only implicitly defined within the whole set of variant parts.

- In addition in many cases existing systems have a lack of powerful and uniform user interface for efficient and comfortable product data management. In particular this is true for small and medium sized companies.

In this paper a concept for integrated rule-based product data management is introduced which compensates the mentioned problems of existing systems in the field of product data exchange and product data management. A primary goal is to integrate data of business oriented applications (e.g. customer individual products, bills of material) and data of technical applications (e.g. routing plans, technical operations) of an enterprise into a central database. In addition, dependencies between variant parts (products) and routing plans as well as dependencies between variant parts and bills of material (BOMs) are transformed into a rule system which is stored within the central database.

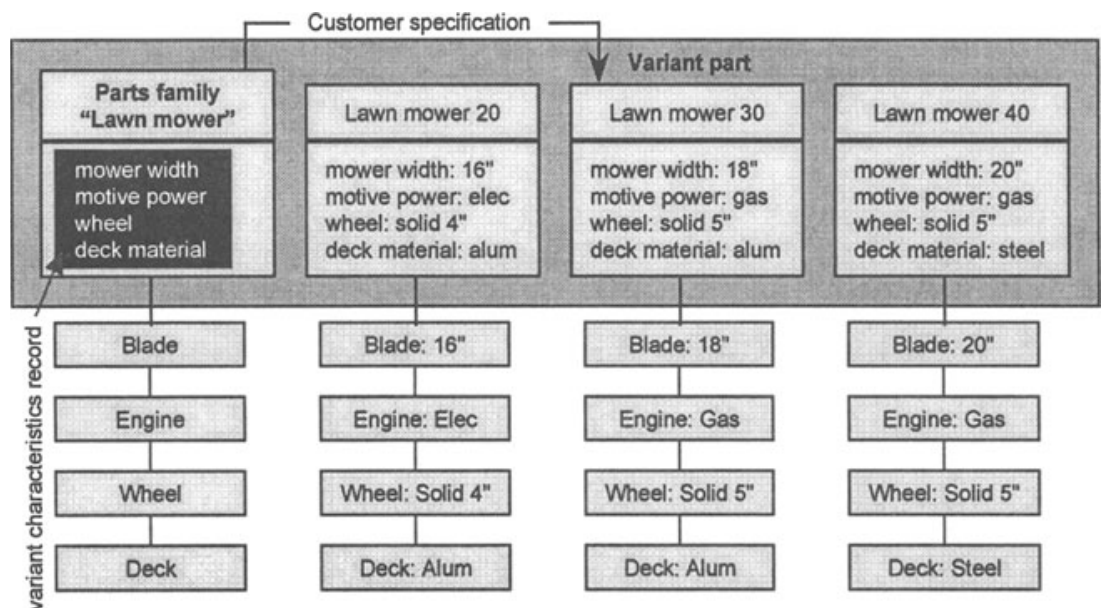

Figure 1-Parts family, variant part and variant characteristics record

A prototype for the administration of product data and the corresponding rule system is already implemented (Wøßß 1998). The development of a powerful and homogeneous graphical user interface for this prototype is a main focus of this paper.

The remainder of the paper is organized as follows: Section 2 starts with a detailed discussion of unsolved problems with the management of product data. In Section 3 the concept of integrated product management is introduced. This section 
includes a description of the system architecture and the main modules of ProMan (Product Data Manager). First, the generator for variant parts and variant BOMs which is responsible for the dynamic generation of variant parts and variant BOMs. Second, the transformation processor for BOM data which is responsible for the dynamic generation of specific types of BOMs according to organizational needs. Third, the routing plan processor which dynamically generates variant depending routing plans. The user interface of ProMan is homogeneous in the sense that it covers all those functions necessary for using each individual ProMan module. Section 4 concludes and gives an outlook on future research.

Before going into detail, the most important terms used in this paper are defined (Figure 1): A variant part or a variant is defined as a customer specific shape, design, or modification of a final product. The customer specifies a set of variant characteristics which describe a variant part. The set of all variant characteristics of a single variant is called variant characteristics record. The variant specification is the set of customer-specified characteristics of a variant part. That means, that the variant specification is the description of a customer specific final product. The parts family is a set of similar parts in the sense of their manufacturing technology and/or construction. Each parts family possesses its own variant characteristics record which is a valid combination of variant characteristics for the parts family at hand.

\section{EXISTING PROBLEMS WITH THE MANAGEMENT OF PRODUCT DATA IN CIM SYSTEMS}

This section gives a survey of general problem areas in the field of product data management:

- data exchange between CAx systems (computer-aided systems),

- administration of a large number of variant parts, variant BOMs, variant routing plans and

- application specific and complex user interfaces.

\subsection{Data exchange between CAx systems}

Today in many enterprises the following scenario can be found out: Different organization units of an enterprise have different structural (semantic) views on the same data. For example, the design engineer creates a construction bill of material (BOM) with the CAD (computer-aided design) system and the operations planner needs a production BOM within the PPS (production planning and control system). The type of a BOM which is created within the CAD system is characterized by geometrical data and functional subassemblies, whereas the production planning system requires a BOM which structures the parts and subassemblies from a production-oriented point of view.

To overcome this structural problems, each software system (CIM component / CAx system) administrates its own specialized database. The drawbacks of this trend are high data redundancies and many inconsistencies between the different databases (Bass 1996). 
Existing solutions try to solve the "data exchange problem" with standardized interfaces between two corresponding CAx systems. A representative example of a standardized interfaces is STEP ISO 10303 (Standart d'Échange et de Transfert) (Brändli 1994, Grabowski 1994, Rembold 1994). But an increasing number of CAx systems results in an increasing number of necessary conversion programs (and/or interfaces). A powerful an efficient data exchange between CAx systems is not possible.

\subsection{Administration of a large number of variant parts}

Beside the lack of powerful data exchange mechanisms between CAx systems the administration of a large number of variant parts, variant BOMs and variant routing plans (RP) is still a problem. In principal, there are two alternatives to administrate variant BOMs and variant RPs (Scheer 1995). A closed administration of variant BOMs and variant RPs requires pre-defined variant parts. Thus, all variant final products are pre-defined which causes many restrictions on the flexibility of the customer specific assembly of final products. Nevertheless, the administration of variants can be done without interaction of the operations planner. But the maintenance of this large amount of variant parts, variant BOMs and RPs is very time and cost intensive. To overcome the conflicts between high flexibility and automatic administration of variants, open administration of variant parts is a feasible compromise. The integration of similar variant parts to a parts family is the most important concept of this principle.

The approach presented in this paper adheres to the principle of open administration of variants since this concept offers more flexibility, in particular for the maintenance of a large number of variants, variant BOMs and variant RPs.

\subsection{Application specific and complex user interfaces}

In most cases existing CIM systems consist of heterogeneous CIM components, each with its own functional concept, with its own database(s) and with a specific user interface. As already mentioned, most of the CIM components store and administrate product data. A significant drawback of this situation is, that all these CIM components have similar user functions for the administration of product data. Therefore beside data redundancy there is also a redundancy of application and/or user functions. Each CIM component is characterized by its own individual user interface. Software users in the field of product data management are often confronted with an intensive training phase before they are able to use a CIM component. Redundant user functions in different CIM components result in redundant training effort and redundant expert (user) knowledge. It is important to consider also the rationalization potential in this field.

\section{INTEGRATED PRODUCT DATA MANAGEMENT}

In networked enterprises the integration of business oriented and technical components in CIM systems often fails because of the lack of powerful data exchange mechanisms between the corresponding software systems. Since CIM 
components store and administrate different types of data but most of them administrate some kind of product data a powerful and integrated management of product data results in a significant improvement of the whole CIM system (Anderl 1997).

For production planning two kinds of product data are very important:

- bills of material specify the material requirements of a product

- routing plans specify the sequence of operations which are necessary to produce a product.

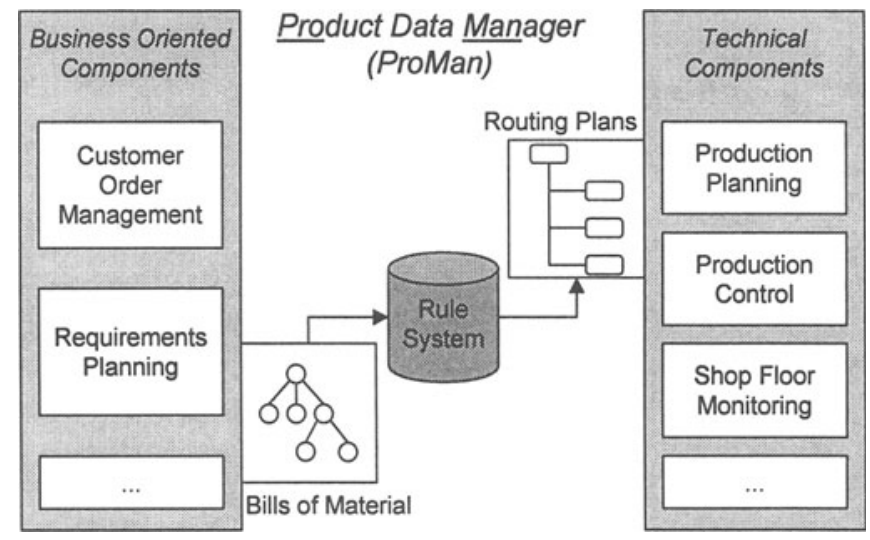

Figure 2 - ProMan: integration of business oriented and technical components

Actual bills of material are derived from a customer specification of a final product. A customer orders a final product with individual design and type (color, size, model, ...). Hence, the required variant of a product is defined. Based on this specification, the variant bill of material of the customer individual product is derived and the material requirements are determined. With this task the process chain of an enterprise changes from business oriented applications to technical applications (Figure 2). In the next step it has to be decided which sequence of operations (routing plan) is necessary to produce the customer individual final product. The specification of dependencies between BOMs and RPs is very important for this decision process. The number of dependencies is decisive for the number of interactions between the operations planner and the CIM software system. If there are only a few dependencies transformed into the CIM software, then frequent technical decisions of the operations planner still are necessary.

To establish a connection between business oriented and technical processes within an enterprise in this paper a rule-based Product Data Manager (ProMan) is introduced which is divided into three major modules:

- The main functionality of the proposed rule-based generator for variants is, that it determines the variant subpart of each subposition of the BOM tree of the final product, based on a customer specified set of final product characteristics. Thus, variant $\mathrm{BOMs}$ are generated dynamically at application runtime and therefore the amount of BOM data is significantly reduced. 
- The rule-based transformation processor for bill of material data creates an individual type of bill of material for each organization unit of an enterprise.

- The rule-based routing plan processor derives a variant specific routing plan from the variant bill of material and the basic routing plan. As well as BOMs, RPs are generated dynamically and therefore the number of stored RPs is also significantly reduced.

In addition ProMan provides an efficient user interface for integrated management of product data.

In contrast to existing systems (Kurbel 1995, Macdonald 1996, Nicolai 1993, Paul 1995), this approach considers dependencies between BOMs and RPs. This concept significantly reduces the amount of product data that has to be administrated, because BOMs and RPs are generated dynamically at application runtime. Moreover, knowledge about production planning is transformed into a rulebase. Since this knowledge then is independent from the operations planner the necessary interaction between the operational experts of an enterprise and the CIM software is reduced. Due to a central administration of product data (variant parts, BOMs, RPs), data exchange between CIM components is significantly improved. Due to an integrated and uniform user interface redundant user functions in different CIM components are prevented and the maintenance of product data is much more efficient.

\subsection{Generator for variant parts and variant BOMs}

In order to establish a rule-based generator for variants and variant BOMs, different variant parts of a product are integrated to a parts family and different variant BOMs are integrated to a basic BOM. The main advantage of this approach is that the variant specific characteristics are separated from the BOM and therefore the number of BOMs is reduced to the number of basic BOMs. The dependencies between the final product and positions of the corresponding subtree are defined by means of "IF-THEN" rules. For example: "If the color of the lawn mower is green then the color of the lawn mower deck has to be green".

The result of the whole generation process is a customer specified final product together with the corresponding variant BOM.

\subsection{Transformation processor for BOM data}

As discussed in Section 1 and 2, a central and integrated management of BOM data is an important step to improve the interaction between CIM components of an enterprise. But this integration process causes special problems, because it is not sufficient to integrate plain BOM data. In addition the specific semantics of BOM data has to be considered. To make the integration of BOM data of all organization units possible, this approach defines several types, e.g. a construction-, production-, order- or delivery BOM as different (structural) views of the same basic BOM. This kind of view differs in quality and complexity from the well-known view concept of relational databases, which does not support recursive algorithms and the specification of rules.

Different types of BOMs are provided by the definition of a set of transformation specifications (set of "IF-THEN" rules) such as: "If the BOM of part A has to be 
transformed into type $T$ then decompose the subtree which is located at position $\mathrm{P}$ ". The transformation specifications are realized as a set of rules which may be defined at each node of the basic BOM, specifying those transformation operations that are necessary to transform a basic BOM into the requested type.

An important advantage of this approach is, that if there are updates of the BOM data necessary, it is sufficient to change the basic BOM and/or the corresponding transformation specifications. Thus, all specific types of BOMs have a consistent state and the updates are immediately valid in all organization units.

\subsection{Routing plan processor}

Similar to the concept of the generator for variant parts and variant BOMs for the rule-based routing plan processor variant-BOM-depending RPs of a parts family are integrated to basic routing plan. The data structures of the basic RP is independent from a customer specific variant part. The basic RP covers and integrates all production instructions for each variant part of a parts family. Different operation instructions for different variants are expressed by alternatives within the sequence of operations of a routing plan.

The procedures of ProMan derive the variant BOM from both, the parts family and the basic BOM by using transformation rules. After this step the rule-based routing plan processor derives a variant specific $R P$ from the variant $B O M$ and the basic $\mathrm{RP}$ by processing the corresponding routing plan rules.

Based on the customer specific variant part the set of alternative operations of a routing plan is reduced to a single element. Hence, the operation at this position (routing plan element) within the routing plan is exactly defined. In the best case, the specified rules are restrictive enough to exactly define each operation within the routing plan. If two alternative operations are absolute equivalent and therefore the system is not able to determine which operation should be taken, the operations planner has to determine interactively which operation is valid.

\subsection{User interface of ProMan}

For a graphical representation of both, BOMs and RPs, a tree structure is suitable. The user interface of ProMan uses a tree structure not only for the representation of BOMs and RPs, but also for the presentation of the program modules and functions. This approach has several particular advantages:

- The main window of ProMan is separated into two sections (Figure 3). The left section is used for navigating through the program structure which is represented by a graphical tree. The right section shows the menus of the program structure as well as product data, like part families, BOMs and RPs, belonging to a selected program menu. This organization of a user interface allows to navigate through the program structure and through data forms at the same time with the same means. A recursive navigation through data and/or program structures, which is an important feature for the management of BOM and RP trees, is also possible.

- Submenus are visible not before a user steps into the menu tree.

- In addition the user interface of ProMan provides five different views on the data and/or program structures. For example this advantage allows a user to handle a 
view on a data structure in one window and a view on program functions or data in another window at the same time.

- The whole system for product data management is based on a uniform user interface.

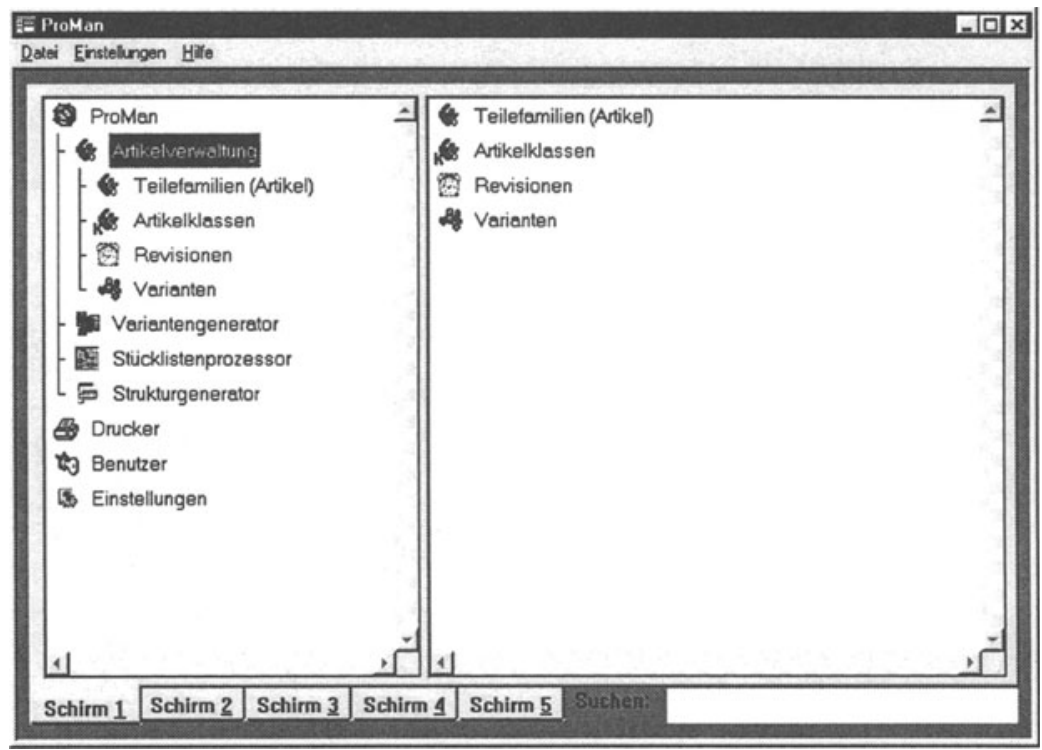

Figure 3 - Main window of ProMan

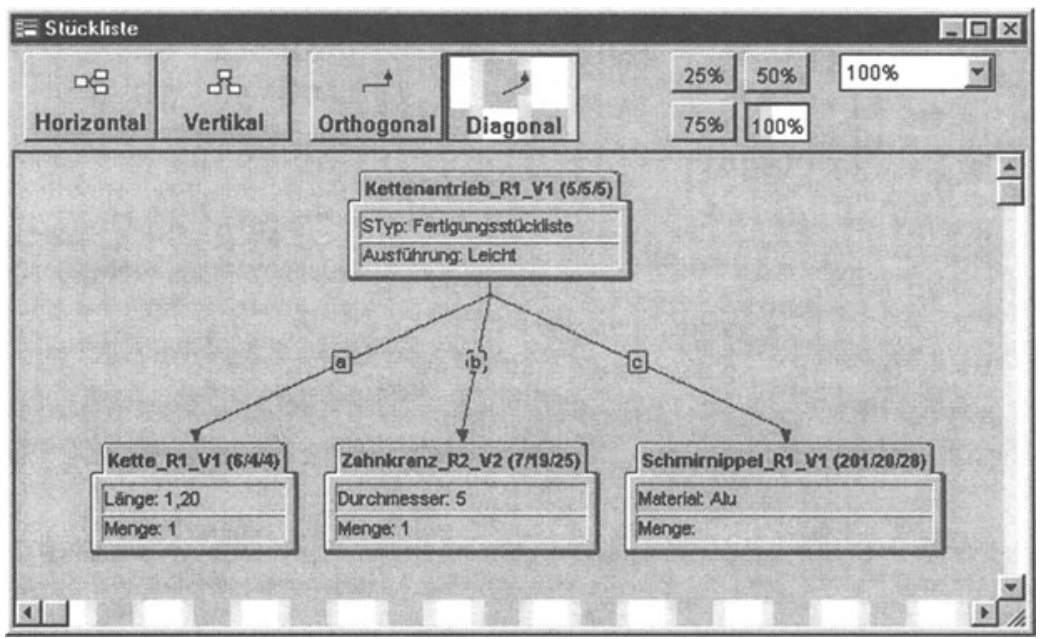

Figure 4-Graphical representation of a bill of material tree

ProMan also allows multi user access, based on the ROWID concept of the database system.

Translation of the terms within the German user interface (Figure 3, Figure 4): Artikelverwaltung...parts management, Teilefamilien (Artikel)...parts families 
(parts), Revisionen...part revisions, Varianten...variant parts, Variantengenerator...generator for variant parts and variant BOMs, Stücklistenprozessor...transformation processor for BOM data, Strukturgenerator...BOM generator, Drucker...printer, Benutzer...user, Einstellungen...options, Stückliste...BOM.

The graphical representation of a BOM tree (Figure 4) provides a zoom function and a left-to-right or top-down orientation of the BOM tree. In addition the edges of the tree appear right-angled or diagonal.

ProMan is implemented with SQLWindows ${ }^{\circledast} 5 . x$ (Gupta $^{T M} /$ Centura $\left.^{T M}\right)$ and it is connected to a SQLBase ${ }^{\circledast}$ 6.x (Gupta $^{\mathrm{TM}}$ / Centura $^{\mathrm{TM}}$ ) database both based on a Microsoft ${ }^{\mathrm{TM}}$ Windows $95 / 98^{\circledR}$ platform.

\section{CONCLUSIONS}

Today many enterprises are still confronted with unsolved problems in the field of product data management. The importance of product data as integration factor between business oriented and technical components of networked enterprises is discussed in Section 1 and 2. In existing systems data exchange and communication between business oriented and technical processes requires many manual user interactions, because most of these systems have no connection between BOMs and RPs.

This paper focuses and provides a solution for several important aspects of product data management:

- Efficient management of a large number of variant parts, variant BOMs and variant specific RPs without redundancy.

- Rule-based management of dependencies between BOM data and RPs and as a consequence an improved integration of business oriented and technical components of a CIM system.

- Powerful data exchange mechanisms for different types of BOMs.

- Expert-independent availability of planning algorithms and planning knowledge by transformation of this knowledge into a central rulebase.

- Efficient management of product data based on an integrated and uniform user interface.

The approach presented in this paper significantly reduces the amount of data that has to be administrated because variant BOMs and variant specific RPs are dynamically generated at application runtime. To realize this concept, dependencies between variant parts, variant BOMs and variant specific RPs as well as knowledge about BOM and RP processing are transformed into a rulebase by means of "IFTHEN" rules.

Due to the advantage of the management of product data, BOMs, RPs and the entire rulebase in a central database, the complexity of data exchange between CIM components is significantly decreased. Specialized interfaces and/or conversion programs are not longer necessary for data exchange. At the same time data redundancy is dramatically reduced. In comparison to existing solutions an improved integration of business oriented and technical components of a CIM system is the consequence. 
In existing CIM systems beside data redundancy there is also a redundancy of application and/or user functions because each CIM component is characterized by its own individual user interface. Users of product data management software often need to be trained intensively before they are able to use a CIM component efficiently. Redundant user functions in different CIM components result in redundant training and redundant expert (user) knowledge. The integrated and uniform user interface for product data management in ProMan compensates this drawbacks. A prototype of ProMan is already implemented.

Further work will be concentrated on the development of concepts for standardized data interchange between different CIM-systems based on Internet/Intranet technology (WWW, extensible markup language). Focus of interest will be EDI (electronic data interchange) between customers and the enterprise as well as cooperation between departments of an enterprise. This will be a further step to improve the integration and efficiency of CIM systems.

\section{REFERENCES}

1. Anderl, R., Katzenmaier, J. "Trends in der Produktdatenverwaltung", Engineering Data Management, in: Industrie Management: Innovative Strategien fur die Produktion, Ed.: Prof. Dr. Hermann Krallmann, Prof. Dr.-Ing. Bernd Scholz-Reiter, GITO-Verlag Berlin, 1996/97.

2. Bass, I. "Product Data Management: Winning the Data Game", CiME Computer Integrated Manufacture \& Engineering, Lionhaert Publishing Inc. USA, Vol. 1/2 1996.

3. Brăndli, N., Nicolai, H., Krzepinski, A. "Modellierung einer CAD/PPS-Schnittstelle", CIMManagement, Ed.: Prof. Dr. Hermann Krallmann, Oldenbourg Verlag Munchen, Vol. 10/1, 1994.

4. Breitenstein, R., Stratmann, J. "Modellierung einer durchgăngigen, variantenreichen Produktstruktur im SAP-System R/2", CIM-Management, Ed.: Prof. Dr. Hermann Krallmann, Oldenbourg Verlag München, Vol. 10/1, 1994.

5. Grabowski, H., Anderl R., Erb, J., Polly, A. "STEP - Grundlage der Produktdatentechnologie; Aufbau und Entwicklungsmethodik", CIM-Management, Ed.: Prof. Dr. Hermann Krallmann, Oldenbourg Verlag Munchen, Vol. 10/4, 1994.

6. Kurbel, K. "Produktions- planung und -steuerung: Methodische Grundlagen von PPS-Systemen und Erweiterungen", Oldenbourg Verlag Munchen, 1995.

7. Macdonald, D. "Beyond PDM: The Next Generation", Computer Integrated Manufacture \& Engineering, Lionhaert Publishing Inc. USA, Vol. 1/2 1996.

8. Nicolai, H., Eulenberger, L., Krzepinski, A. "Modellierung einer CAD/PPS-Schnittstelle", CIMManagement, Ed.: Prof. Dr. Hermann Krallmann, Oldenbourg Verlag München, Vol. 9/4, 1993.

9. Paul, G., Wierschin, H., Paul, R., Hofmann, M. "Produktdatenverwaltungssysteme - Methoden und Werkzeuge zur Unternehmensintegration", CIM-Management, Ed.: Prof. Dr. Hermann Krallmann, Oldenbourg Verlag Munchen, Vol. 11/4, 1995.

10. Poensgen, W. "Prozeßorientierter Losungsansatz fur die Integration von Vertrieb und PPS bei variantenreichen Produkten", CIM-Management, Ed.: Prof. Dr. Hermann Krallmann, Oldenbourg Verlag München, Vol. 10/1, 1994.

11. Rembold, U., Nnaji, BO., Storr, A. "CIM: Computeranwendung in der Produktion", AddisonWesley, 1994.

12. Scheer, A. "Wirtschaftsinformatik, Referenzmodelle fur industrielle Geschåtprozesse", Springer Verlag Berlin, Heidelberg, New York, $6^{\text {th }}$ edition, 1995.

13. Thomas, R. "Varianten - Herausforderung fur Fertigungsunternehmen", ZWF Zeitschrift fur wirtschaftiche Fertigung und Automatisierung, Ed.: Prof. Dr. Gunter Spur, Carl Hanser Verlag Munchen, Vol. 87/11, 1992.

14. WoB, W., Wagner R.: "Rule-based Management of Product Data in CIM Systems", in: Intelligent Systems for Manufacturing, Multi-Agent Systems and Virtual Organizations, Proceedings of the BASYS'98 - 3rd IEEE/IFIP International Conference on Information Technology for Balanced Automation Systems in Manufacturing, Prague 1998, Eds. Camarinha-Matos L M., Afsarmanesh H., Marik V., Kluwer Academic Publishers, The Netherlands, 1998. 